\title{
Partial Nephrectomy is the Surgical Treatment of Choice for (Most) Complex Localized Renal Tumors
}

\author{
Annika Herlemann ${ }^{\mathrm{a}, \mathrm{b}}$, Anobel Y. Odisho ${ }^{\mathrm{a}}$ and Sima P. Porten ${ }^{\mathrm{a}, *}$ \\ ${ }^{a}$ Department of Urology, University of California, San Francisco, Helen Diller Family Comprehensive Cancer \\ Center, San Francisco, CA, USA \\ ${ }^{\mathrm{b}}$ Department of Urology, Ludwig-Maximilians-University of Munich, Munich, Germany
}

Keywords: Partial nephrectomy, nephron-sparing surgery, kidney cancer, renal tumors

The decision to undergo partial nephrectomy (PN) versus radical nephrectomy $(\mathrm{RN})$ for a localized renal mass should take various factors into consideration, including tumor characteristics, patient's health status and preference, and risks and benefits of these each approach. PN represents a suitable surgical approach for small renal tumors in favorable locations. It may also better preserve long-term renal function, particularly in patients who may need surgery for a contralateral kidney mass or have preexisting chronic kidney disease. Conversely, RN may be preferred in elderly patients with severe (non-renal related) comorbidities, in patients with an anatomically challenging tumor location, or for larger or locally advanced masses.

The presentation and management of renal tumors has changed substantially during the last few decades. The increasing use and quality of crosssectional imaging has been associated with increased incidence, earlier detection and improved staging of renal masses [12]. Most kidney tumors are now diagnosed incidentally in asymptomatic patients [3]. In a recent study by Welch et al. examining a large cohort of Medicare beneficiaries, increase rates of computed tomography (CT) imaging was associated with

\footnotetext{
${ }^{*}$ Correspondence to: Sima P. Porten, MD, MPH, 550 16th Street, Sixth Floor, UCSF Box 1695, San Francisco, CA 94143, USA. Tel.: +1 415885 3695; Fax: +1 415476 5366; E-mail: sima.porten@ucsf.edu.
}

a higher risk of nephrectomy, presumably from incidental detection of renal masses, with little change in morality [4]. The majority of these patients show small, localized tumors (T1) and the demand for nephron-sparing surgery is high, as stage predicts favorable survival outcomes for these patients [5]. The indication to perform $\mathrm{PN}$ has been expanded and $\mathrm{RN}$ is no longer the surgical treatment of choice for small, localized renal masses as reflected by current international guidelines [3,6]. Specifically, based on available oncologic and quality of life data, PN should be recommended over RN for cT1b tumors if technically possible, irrespective of the surgical approach $[3,6]$. Laparoscopic radical nephrectomy (RN) should be offered to patients with locally advanced tumor growth or in T1 tumors with unfavorable location [6].

There is no debate among urologists in using PN for most clinical T1a masses. This is especially true in contemporary practice as experience in partial nephrectomy grows, primarily driven by the rise of robotic minimally-invasive surgery. With a relatively favorable learning curve ( 25 cases), dissemination of robotic assisted technology and has led to increased use of partial nephrectomy, from $8.6 \%$ in 2000 to $27 \%$ in 2011 [7]. The increase of partial nephrectomies performed was attributable to robotic assisted surgery that increased by $15 \%$, with a concomitant decrease of open surgery by $33 \%$ [7]. With this experience, 
surgeons have naturally started to push the envelope for partial nephrectomy to more complex cT1a tumors, and larger masses (cT1b and T2 tumors). Appropriately, this is necessary for patients with a solitary kidney, hereditary syndrome, known bilateral tumors, and significant chronic renal disease. Conversely, in patients with significant comorbidity, frailty, and limited life expectancy, active surveillance may be considered $[3,6]$. The area of controversy and debate is in the optimal management of patients with cT1b and T2 tumors, normal renal function, and a normal contralateral kidney, and is the focus of the current article [6, 8]. Shared goals between patients and providers include cure (both of local disease and to prevent metastatic disease), expose patients to minimal morbidity (surgical in the short term, medical in the long term), and maximize renal function. Surgeons want to provide every patient the very best personal outcome. Is pushing the envelope with PN necessary to meet that goal? We believe, for most patients in this controversial clinical situation, partial nephrectomy is a necessary consideration.

\section{GOAL \#1: OPTIMIZE ONCOLOGIC OUTCOMES}

EORTC 30904 is the only prospective randomized control trial comparing PN to RN and established the oncologic equivalence between the two treatment modalities in masses $<5 \mathrm{~cm}$ for cancer specific survival. However, there was an overall survival benefit favoring RN, and it has been criticized for shortcomings such as being underpowered and that PN was performed by low volume surgeons. For larger tumors, only retrospective data is available, with accompanying selection bias (patients on average 2-3 years younger and with less comorbidity undergoing partial nephrectomy) [9]. However, multiple institutional and population-based analyses (both historical and contemporary) have reported that PN provides adequate cancer control equivalent to $\mathrm{RN}$ for cT1b tumors and recent data suggest that PN for T2 tumors is not associated with reduced cancer specific survival [10-18]. Mir et al., performed a timely systematic review and meta-analysis of over 11,000 patients in 21 studies comparing PN with $\mathrm{RN}$ in patients with $\mathrm{cT} 1 \mathrm{~b}$ and $\mathrm{T} 2$ renal tumors [19]. They found that the likelihood for cancer specific mortality was lower for PN (OR 0.58, 95\% CI $0.41-0.81 ; p=0.001)$, even when the analysis was restricted to cT2 tumors (OR $0.65,95 \% \mathrm{CI}$ $0.44-0.97 ; p=0.03$ ). They also reported that local and distant recurrence rates were lower in the PN group than the RN group (OR $0.6095 \%$ CI $0.46-0.79$; $p<0.001$ ).

Traditionally, the presence of a positive surgical margin (PSM) has been considered a risk factor for disease recurrence and is cited as a drawback to pushing the boundary with PN [20]. Due to technical improvements and greater use of PN, PSM incidence has become less common and is reported in up to $8 \%$ of patients [21, 22]. In terms of surgical approach, rates of PSMs vary slightly and occur in $7.0 \%$ of patients after open $\mathrm{PN}, 8.1 \%$ after laparoscopic PN, and $8.7 \%$ after robot-assisted PN [21, 23]. However, not all PSMs in kidney cancer patients lead to recurrence; therefore, there is no clear recommendation in terms of further management of the affected kidney [20]. Long-term analyses suggest that margins $<5 \mathrm{~mm}$ or simple tumor enucleation both show similarly favorable oncologic outcomes at followup $[24,25]$. The thickness of healthy parenchyma surrounding the tumor does not appear to be important as long as complete resection is attained, and may lead to decrease in future renal function [23]. Intraoperative frozen section to evaluate surgical margins during PN is of minor importance; the surgeon's macroscopic assessment of negative margins does provide reliable results [23]. PSM has no impact on metastatic-free, cancer-specific or overall survival compared to patients with a negative surgical margin (NSM) [20, 22], even in with stage T1bT3 tumors $>4 \mathrm{~cm}$ [26]. Although the presence of PSM is associated with more recurrences, survival rates of NSM patients and PSM patients after PN for a localized tumor are equivalent [20]. Therefore, in considering surgical options for patients with locally advance disease, PN offers effective cancer control based on a large body of comparative evidence.

\section{GOAL \#2: MINIMIZE SURGICAL MORBIDITY}

PN is generally considered a more technically complex approach that is more time-consuming and may be associated with an increased rate of surgical complications [1, 27]. The risk for common perioperative and postoperative complications such as bleeding (relative risk (RR) 1.94) and urinary fistula (RR 20.92) was significantly increased for PN 
compared to RN, with no difference in re-operation rates in a recent Cochrane review including all stages of disease [28]. Supporting these findings, in a systematic review focusing on $\mathrm{pT} 1 \mathrm{~b}$ and $\mathrm{T} 2$ tumors, the likelihood of postoperative complications was higher for the PN group (OR 1.74). In the subset of cT2 patients, the likelihood of complications was higher (RR 2.0) as was estimated blood loss (WMD $107.6 \mathrm{ml})$.

It is not surprising that complication rates are higher as the complexity of surgical operations increase. Furthermore, prospective data proposed that increasing tumor anatomic complexity (not just size) quantitated using the nephrometry (RENAL) score, was associated with the development of major complications within 30 days after PN using the Clavien-Dindo classification (OR 5.4) [29, 30]. Minor complications were similar between groups regardless of tumor complexity [30]. As with any high-risk surgical procedure, these complication rates need to be taken into context to possible benefit to the patient (as discussed in the next section). It does not appear that size alone is the entire story. Rather than eliminating partial nephrectomy as a consideration for patients with cT1 and T2 disease due to the possibility of increased complications, the focus should be on addressing and mitigating all possible contributors to risk. As with any surgical endeavor treatment decisions and approach should be made in the context of comprehensive surgical assessment (tumor factors, surgeon factors, and patient factors including patient preferences).

\section{GOAL \#3: PROTECT RENAL FUNCTION}

The expected rationale for expanding the use of PN would be to preserve renal function (which in turn, would prevent downstream morbidity) if oncologic outcome is equivalent to RN [9]. As discussed above, this benefit comes with the risk of increased surgical complications. This goal is often the crux of the PN vs RN debate and the subject of heated discussion: Does preservation of renal function have a true impact in reducing long term morbidity (CKD, cardiovascular events, dialysis) and overall mortality and should it be done in exchange for a higher rate of short-term complications? [31]

Initially, literature from other specialties reported that patients with CKD and estimated glomerular filtration rate $(\mathrm{eGFR})<60 \mathrm{ml} / \mathrm{min} / 1.73 \mathrm{~m}^{2}$ had significantly higher risks of cardiovascular events (HR
1.4) and of all-cause death (HR 1.2) than patients with no renal impairment $[32,33]$. These risks were even higher in patients with a eGFR $<30 \mathrm{ml} / \mathrm{min} / 1.73$ $\mathrm{m}^{2}$ (HR 2.8 and HR 3.2, for cardiovascular events and all-cause death, respectively) [32,33]. Following these reports, single institution and population-based analyses suggested that reduced renal function in $\mathrm{RN}$ patients compared with PN patients was associated with worse overall survival. The largest of these studies was by Weight et al., who reported outcomes for approximately $1,000 \mathrm{cT} 1 \mathrm{~b}$ patients and found that loss of renal function after RN was associated with $25 \%$ higher cardiovascular mortality and $17 \%$ overall mortality [34]. EORTC 30904 provides the only prospective randomized controlled data (albeit for masses $5 \mathrm{~cm}$ or less) looking at renal function and mortality in patients who underwent PN vs RN. PN compared with RN was associated with a $21 \%$ reduction in the absolute risk of at least moderate renal dysfunction $\left(e G F R<60 \mathrm{ml} / \mathrm{min} / 1.73 \mathrm{~m}^{2}\right.$ ). In the intention-to-treat population this did not translated into a mortality benefit, and conversely PN showed a significantly decreased 10 -year overall survival (OS) compared to RN (75.5 vs. $81.1 \%$ ). However, in the targeted population of renal cell cancer patients, the OS trend in favor of $\mathrm{RN}$ was no longer significant [9].

These unexpected findings have been explained in a variety of ways: study design, accrual, historical cohort managed differently than contemporary cohorts, and biologically- not all renal dysfunction is equivalent. Recent studies have suggested that long term progressive decline in renal function per year is more profound in patients with medical renal disease compared with surgical renal disease (5\% vs $0.7 \%$ ) [31]. In the contemporary meta-analyses by Mir et al., patients with clinical $\mathrm{T} 1 \mathrm{~b}$ and $\mathrm{T} 2$ tumors treated with PN had better postoperative renal function, as demonstrated by higher postoperative eGFR (weighted mean difference (WMD) $12.4 \mathrm{ml} / \mathrm{min}$ ), lower likelihood of postoperative onset of chronic kidney disease (RR 0.36), lower decline in eGFR (WMD $-8.6 \mathrm{ml} / \mathrm{min}$ ), and improved all-cause mortality (OR 0.67) [19]. Overall, the data supporting preserving renal function is compelling and should not be lightly dismissed, despite potential bias in the currently available studies. The understanding of meaningful renal dysfunction and risk of development of renal dysfunction from surgery, existing comorbidities, and protecting nephrons due to potential renal deterioration from development of comorbidities is still evolving, and further studies will hopefully improve our knowledge and treatment decisions. 


\section{GOAL \#4: PRESERVE QUALITY OF LIFE}

There is limited data with regard to patient reported outcomes after treatment with PN or RN for $\mathrm{cT} 1 \mathrm{~b}$ and $\mathrm{T} 2$ tumors, with the majority of studies including patients with smaller masses. In one prospective study, PN patients reported better quality of life (QoL), improving with time, in many aspects of QoL as measured by validated questionnaires, such as SF36 and EORTC QLQ-C30 [35, 36]. Patients after $\mathrm{RN}$ reported an increased fear [35, 36] associated with living with a solitary kidney, but also less concern about of cancer recurrence. In general, patients with tumors $<4 \mathrm{~cm}$ and a normal contralateral kidney showed the highest overall QoL scores and superior recovery of stress from cancer after treatment $[35,36]$ as expected. In a retrospective analysis, the mean mental and physical component SF-36 scores were similar between the PN and RN groups. However, patients (regardless of the type of surgery) who had higher complication rates showed significantly lower QoL scores in the general health domain, which may temper any anxiety related benefit $[35,37]$. Partial nephrectomy may the correct choice for some patients from a QoL perspective based on personal preferences; however, complications will negatively affect patient reported outcomes.

In a perfect world, we should be designing an RCT for this patient population, using a contemporary cohort and technology, patient reported outcomes, and modern renal function assessment. However, realistically, this trial will never accrue and in its place, we have our imperfect literature riddled with heterogeneity and bias. In the spirit of this article defending and advocating the use of partial nephrectomy for cT1b and T2 tumors we support partial nephrectomy for most patients. However, in this time of exciting progress and rapidly improving technology we need to be mindful that our treatment decisions embody our common goals and our oath to "first do no harm." Our research and debates need to evolve past just PN vs RN and move toward improved risk-stratification and truly personalized, multidisciplinary treatment plans.

\section{FUNDING SOURCES}

None

\section{CONFLICT OF INTEREST}

None

\section{REFERENCES}

[1] Van Poppel H, Da Pozzo L, Albrecht W, et al. A prospective randomized EORTC intergroup phase 3 study comparing the complications of elective nephronsparing surgery and radical nephrectomy for low-stage renal cell carcinoma. Eur Urol. 2007;51(6):1606-15. doi:10.1016/j.eururo.2006.11.013

[2] Huang WC, Atoria CL, Bjurlin M, et al. Management of Small Kidney Cancers in the New Millennium: Contemporary Trends and Outcomes in a Population-Based Cohort. JAMA Surg. 2015;150(7):66472. doi:10.1001/jamasurg.2015.0294

[3] Campbell S, Uzzo RG, Allaf ME, et al. Renal Mass and Localized Renal Cancer: AUA Guideline. J Urol. 2017;198(3):520-9. doi:10.1016/j.juro.2017.04.100

[4] Welch HG, Skinner JS, Schroeck FR, Zhou W, Black WC. Regional Variation of Computed Tomographic Imaging in the United States and the Risk of Nephrectomy. JAMA Intern Med. 2018;178(2):221. doi:10.1001/jamainternmed.2017.7508

[5] Kane CJ, Mallin K, Ritchey J, Cooperberg MR, Carroll PR. Renal cell cancer stage migration: Analysis of the National Cancer Data Base. Cancer. 2008;113(1):78-83. doi: $10.1002 /$ cncr. 23518

[6] Ljungberg B, Cowan NC, Hanbury DC, et al. EAU guidelines on renal cell carcinoma: The 2010 update. Eur Urol. 2010;58(3):398-406. doi:10.1016/j.eururo.2010.06.032

[7] Patel HD, Mullins JK, Pierorazio PM, et al. Trends in renal surgery: Robotic technology is associated with increased use of partial nephrectomy. J Urol. 2013;189(4):1229-35. doi:10.1016/j.juro.2012.10.024

[8] Lee HJ, Liss MA, Derweesh IH. Outcomes of partial nephrectomy for clinical $\mathrm{T} 1 \mathrm{~b}$ and $\mathrm{T} 2$ renal tumors. Curr Opin Urol. 2014;24(5):448-52. doi:10.1097/MOU.0000000000000081

[9] Van Poppel H, Da Pozzo L, Albrecht W, et al. A Prospective, Randomised EORTC Intergroup Phase 3 Study Comparing the Oncologic Outcome of Elective NephronSparing Surgery and Radical Nephrectomy for Low-Stage Renal Cell Carcinoma. Eur Urol. 2011;59(4):543-52. doi:10.1016/j.eururo.2010.12.013

[10] Crépel M, Jeldres C, Perrotte P, et al. Nephronsparing surgery is equally effective to radical nephrectomy for T1BNOM0 renal cell carcinoma: A population-based assessment. Urology. 2010;75(2):271-5. doi:10.1016/j.urology.2009.04.098

[11] Badalato GM, Kates M, Wisnivesky JP, Choudhury AR, McKiernan JM. Survival after partial and radical nephrectomy for the treatment of stage T1bN0M0 renal cell carcinoma (RCC) in the USA: A propensity scoring approach. BJU Int. 2012;109(10):1457-62. doi:10.1111/j.1464-410X.2011.10597.x

[12] Meskawi M, Becker A, Bianchi M, et al. Partial and radical nephrectomy provide comparable long-term cancer control for T1b renal cell carcinoma. Int J Urol. 2014;21(2):122-8. doi:10.1111/iju.12204

[13] Hansen J, Sun M, Bianchi M, et al. Assessment of cancer control outcomes in patients with high-risk renal cell carcinoma treated with partial nephrectomy. Urology. 2012;80(2):347-53. doi:10.1016/j.urology.2012.04.043

[14] Kopp RP, Mehrazin R, Palazzi KL, et al. Survival outcomes after radical and partial nephrectomy for clinical $\mathrm{T} 2$ renal tumours categorised by R.E.N.A.L. nephrometry score. BJU Int. 2014;114(5):708-18. doi:10.1111/bju.12580 
[15] Long CJ, Canter DJ, Kutikov A, et al. Partial nephrectomy for renal masses $\geq 7 \mathrm{~cm}$ : Technical, oncological and functional outcomes. BJU Int. 2012;109(10):1450-6. doi:10.1111/j.1464-410X.2011.10608.X

[16] Maddox M, Mandava S, Liu J, Boonjindasup A, Lee BR. Robotic partial nephrectomy for clinical stage T1b tumors: Intermediate oncologic and functional outcomes. Clin Genitourin Cancer. 2015;13(1):94-9. doi:10.1016/j.clgc.2014.07.011

[17] Joniau S, Vander Eeckt K, Srirangam SJ, Van Poppel $\mathrm{H}$. Outcome of nephron-sparing surgery for T1b renal cell carcinoma. BJU Int. 2009;103(10):1344-8. doi:10.1111/j.1464-410X.2008.08230.x

[18] Leibovich BC, Blute ML, Cheville JC, Lohse CM, Weaver AL, Zincke H. Nephron sparing surgery for appropriately selected renal cell carcinoma between 4 and $7 \mathrm{~cm}$ results in outcome similar to radical nephrectomy. J Urol. 2004;171(3):1066-70. doi:10.1097/01.ju.0000113274.40885.db

[19] Mir MC, Derweesh I, Porpiglia F, Zargar H, Mottrie A, Autorino R. Partial Nephrectomy Versus Radical Nephrectomy for Clinical T1b and T2 Renal Tumors: A Systematic Review and Meta-analysis of Comparative Studies. Eur Urol. 2017;71(4):606-17. doi:10.1016/j.eururo.2016.08.060

[20] Bensalah K, Pantuck AJ, Rioux-Leclercq N, et al. Positive surgical margin appears to have negligible impact on survival of renal cell carcinomas treated by nephron-sparing surgery. Eur Urol. 2010;57(3):466-71. doi:10.1016/j.eururo.2009.03.048

[21] Tabayoyong W, Abouassaly R, Kiechle JE, et al. Variation in Surgical Margin Status by Surgical Approach among Patients Undergoing Partial Nephrectomy for Small Renal Masses. J Urol. 2015;194(6):1548-53. doi:10.1016/j.juro.2015.06.076

[22] Yossepowitch O, Thompson RH, Leibovich BC, et al. Positive surgical margins at partial nephrectomy: Predictors and oncological outcomes. J Urol. 2008;179(6):2158-63. doi:10.1016/j.juro.2008.01.100

[23] Marszalek M, Carini M, Chlosta P, et al. Positive surgical margins after nephron-sparing surgery. Eur Urol. 2012;61(4):757-63. doi:10.1016/j.eururo.2011.11.028

[24] Sutherland SE, Resnick MI, Maclennan GT, Goldman HB. Does the size of the surgical margin in partial nephrectomy for renal cell cancer really matter? J Urol. 2002;167(1):61-4. http://www.ncbi.nlm.nih.gov/pubmed/11743276. Accessed May 10, 2018.

[25] Carini M, Minervini A, Lapini A, Masieri L, Serni S. Simple enucleation for the treatment of renal cell carcinoma between 4 and $7 \mathrm{~cm}$ in greatest dimension: Progression and long-term survival. J Urol. 2006;175(6):2022-6; discussion 2026. doi:10.1016/S0022-5347(06)00275-8

[26] Simmons MN, Weight CJ, Gill IS. Laparoscopic radical versus partial nephrectomy for tumors $>4 \mathrm{~cm}$ :
Intermediate-term oncologic and functional outcomes. Urology. 2009;73(5):1077-82. doi:10.1016/j.urology.2008. 11.059

[27] Tomaszewski JJ, Smaldone MC, Uzzo RG, Kutikov A. Is radical nephrectomy a legitimate therapeutic option in patients with renal masses amenable to nephron-sparing surgery? BJU Int. 2015;115(3):357-63. doi:10.1111/bju.12696

[28] Kunath F, Schmidt S, Krabbe L-M, et al. Partial nephrectomy versus radical nephrectomy for clinical localised renal masses. Cochrane database Syst Rev. 2017;5:CD012045. doi:10.1002/14651858.CD012045.pub2

[29] Haifler M, Ristau BT, Higgins AM, et al. External Validation of Contact Surface Area as a Predictor of Postoperative Renal Function in Patients Undergoing Partial Nephrectomy. J Urol. 2017;199(3):649-54. doi:10.1016/j.juro.2017.09.070

[30] Simhan J, Smaldone MC, Tsai KJ, et al. Objective measures of renal mass anatomic complexity predict rates of major complications following partial nephrectomy. Eur Urol. 2011;60(4):724-30. doi:10.1016/j.eururo.2011.05.030

[31] Mir MC, Pavan N, Parekh DJ. Current Paradigm for Ischemia in Kidney Surgery. J Urol. 2016;195(6):1655-63. doi:10.1016/j.juro.2015.09.099

[32] Li L, Lau WL, Rhee CM, et al. Risk of chronic kidney disease after cancer nephrectomy. Nat Rev Nephrol. 2014;10(3):135-45. doi:10.1038/nrneph.2013.273

[33] Go AS, Chertow GM, Fan D, McCulloch CE, Hsu C. Chronic kidney disease and the risks of death, cardiovascular events, and hospitalization. N Engl J Med. 2004;351(13):1296-305. doi:10.1056/NEJMoa041031

[34] Weight CJ, Larson BT, Fergany AF, et al. Nephrectomy Induced Chronic Renal Insufficiency is Associated With Increased Risk of Cardiovascular Death and Death From Any Cause in Patients With Localized cT1b Renal Masses. J Urol. 2010;183(4):1317-23. doi:10.1016/j.juro.2009.12.030

[35] MacLennan S, Imamura M, Lapitan MC, et al. Systematic Review of Perioperative and Quality-oflife Outcomes Following Surgical Management of Localised Renal Cancer. Eur Urol. 2012;62(6):1097-17. doi:10.1016/j.eururo.2012.07.028

[36] Poulakis V, Witzsch U, de Vries R, Moeckel M, Becht E. Quality of life after surgery for localized renal cell carcinoma: Comparison between radical nephrectomy and nephron-sparing surgery. Urology. 2003;62(5):814-20. http://www.ncbi.nlm.nih.gov/pubmed/14624900. Accessed May 10, 2018.

[37] Gratzke C, Seitz M, Bayrle F, et al. Quality of life and perioperative outcomes after retroperitoneoscopic radical nephrectomy (RN), open $\mathrm{RN}$ and nephronsparing surgery in patients with renal cell carcinoma. BJU Int. 2009;104(4):470-5. doi:10.1111/j.1464410X.2009.08439.x 